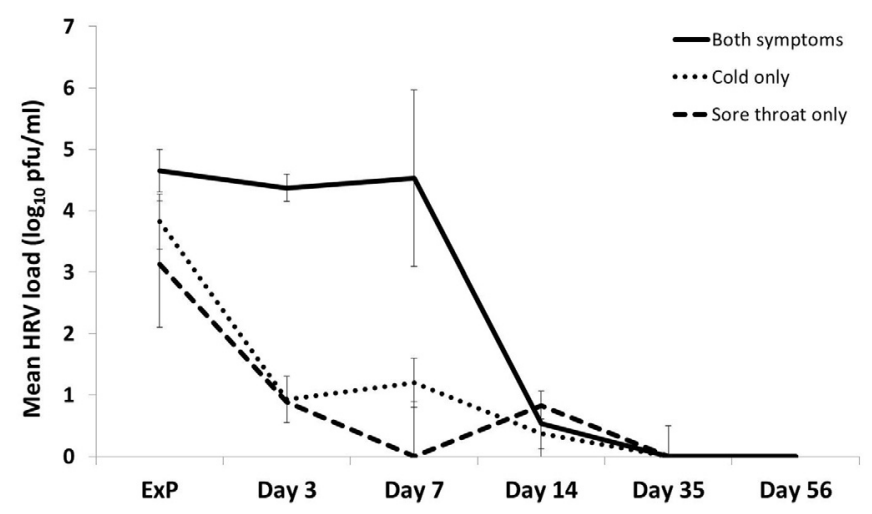

Abstract P68 Figure 1 HRV load during the time-course of an exacerbation and recovery period in sputum samples $(n=106)$ associated with both cold symptoms and a sore throat $(n=23)$, cold symptoms only $(n=67)$ or a sore throat only $(n=16)$. Data shown as mean $( \pm$ SEM)

Introduction Human rhinoviruses (HRV) are the main aetiological agents of virus-associated COPD exacerbations (Seemungal et al. AJRCCM 2001). The relationship between upper respiratory tract symptoms and HRV load at exacerbation presentation $(\mathrm{ExP})$ and during recovery has not been described in naturallyoccurring exacerbations. We quantified changes in airway HRV load at ExP and during the recovery period, in patients reporting symptoms of a cold, sore throat, or both.

Methods Patients in the London COPD cohort recorded new or increased respiratory symptoms on daily diary cards. Exacerbations were defined as an increase in respiratory symptoms for two consecutive days, with at least one symptom being major (dyspnoea, sputum purulence or volume) and the other a major or minor (wheeze, cold, sore throat, cough). Reverse-transcription quantitative PCR was used to detect and quantify HRV in 106 sputum samples collected at $\operatorname{ExP}(\mathrm{n}=38)$ and days 3 $(\mathrm{n}=16), 7(\mathrm{n}=27), 14(\mathrm{n}=16), 35(\mathrm{n}=6)$ and $56(\mathrm{n}=3)$ following.

Results HRV load decreased significantly from ExP to Day 3 in samples associated with either cold symptoms $(\mathrm{p}<0.001)$ or sore throat $(\mathrm{p}=0.049)$ but not in those associated with both symptoms (Figure 1). At Day 3 the HRV load in samples with both symptoms $(\mathrm{n}=5)$ was significantly higher than in those with cold symptoms only $(\mathrm{n}=9)\left(10^{4.22(3.94-4.88)}\right.$ vs $10^{0.55(0-}$ 1.98) $\mathrm{pfu} / \mathrm{ml} ; \mathrm{p}=0.002$ ) but not for those with a sore throat only $(\mathrm{n}=2)\left(10^{0.89(0-0.89)} \mathrm{pfu} / \mathrm{ml} ; \mathrm{p}=0.095\right)$. At Day 7 , the median (IQR) HRV load in samples with both symptoms $(\mathrm{n}=4)\left(10^{4.48(1.85-7.28)} \mathrm{pfu} / \mathrm{ml}\right)$ was significantly higher than in those with cold symptoms only $(\mathrm{n}=19)\left(10^{0(0-2.74)} \mathrm{pfu} / \mathrm{ml} ; \mathrm{p}\right.$ $=0.018)$ or a sore throat only $(\mathrm{n}=4)\left(10^{0(0-0)} \mathrm{pfu} / \mathrm{ml}\right.$; $\mathrm{p}=0.029)$. There was no significant difference at subsequent time-points.

Conclusion Patients reporting both cold symptoms and a sore throat as part of a COPD exacerbation had higher HRV loads than those with just one symptom until after Day 7 post-exacerbation. In patients with both symptoms, the HRV load remained higher for a longer period of time than in patients with only one symptom, which may suggest longer recovery times for more symptomatic patients. These results may inform the timing of administration of antiviral therapies at COPD exacerbation.

\section{Improving lung cancer outcomes}

\section{P69 A STUDY OF THE EFFECT OF THE 2013 'BE CLEAR ON LUNG CANCER' CAMPAIGN ON 2 WEEK WAIT REFERRALS TO AN INNER NORTH WEST LONDON CANCER CENTRE}

HJ Ramsey, YFG Chan, S Obaidee, EF Bowen, SL Elkin. Imperial College Healthcare NHS Trust, London, UK

\subsection{6/thoraxjnl-2014-206260.210}

Introduction The first national 'Be Clear on Cancer' lung cancer campaign ran for 6 weeks from May 2012 with the message 'Been coughing for 3 weeks? Tell your doctor'. During this time there was a $32 \%$ increase in 2 week wait ( 2 WW) referrals, with approximately 700 additional cancers diagnosed compared to 2011.

Method We studied the effect of the 6 week campaign in 2013 on 2 WW referrals to Imperial College NHS trust, comparing with referrals in the 6 week period prior to the campaign. We assessed quality of the referral based on completeness of the 2 WW proforma (scored out of 10), and the outcome of the referral. Direct radiology referrals were not included.

Results The campaign period was 2nd July to mid-August 2013. We studied from 15th May until 15th August 2013. Our referrals increased by $52 \%$ during the campaign ( 25 vs 38 ). The referral quality was unchanged (average score 6.24 pre-campaign and 6.65 during the campaign, $\mathrm{p}=0.41$ ). The proforma was used in $20 / 25$ referrals pre-campaign and 30/38 during the campaign. Table 1 shows the results of the patient information section. Patients received less information during the campaign $(p=n s)$.

Diagnoses There were more referrals diagnosed with lung cancer pre-campaign than during it $(37.5 \%$ vs $13.9 \% \mathrm{p}=0.055)$. One patient in the campaign group was diagnosed with lymphoma. The pre-campaign group had normal investigations in $16.7 \%$ patients, with other diagnoses made in $45.8 \%$ compared to the campaign group which had $22.2 \%(\mathrm{p}=0.6)$ and $61.1 \%$ $(\mathrm{p}=0.25)$ respectively.

There was no significant increase in referrals with a cough as the only symptom $(7 / 25$ vs $11 / 38 p=0.95)$.

In the campaign group, in patients diagnosed with lung cancer, we found a significant improvement in referral score compared to those without cancer ( 8 vs $4.87, \mathrm{p}=0.01$ ). There was no change in the pre-campaign group.

Conclusion Our 2 WW referrals increased during the campaign but fewer patients were diagnosed with lung cancer and more received a non-cancer diagnosis. During the campaign, referral forms for those without cancer were poorly completed which may represent pressure on GPs to refer coughs through the 2 WW pathway despite low suspicion.

\begin{tabular}{|c|c|c|c|}
\hline & 6 weeks pre-campaign & 6 weeks of campaign & $P$ value \\
\hline \multicolumn{4}{|l|}{$\begin{array}{l}\text { No. patients told } \\
\text { that cancer was }\end{array}$} \\
\hline being investigated & $6 / 20(30 \%)$ & $13 / 30(20 \%)$ & 0.34 \\
\hline \multicolumn{4}{|l|}{ No. patients given } \\
\hline \multicolumn{4}{|l|}{$2 W W$ information } \\
\hline leaflet (if on form) & $4 / 17(23.5 \%)$ & $6 / 24(16.7 \%)$ & 0.61 \\
\hline \multicolumn{4}{|c|}{ No. patients told they'd } \\
\hline be seen in 2 weeks & $12 / 20(60 \%)$ & $19 / 30(40 \%)$ & 0.82 \\
\hline
\end{tabular}

